\title{
The utility of surrogate markers in predicting HLA alleles associated with adverse drug reactions in Vietnamese
}

\author{
Dinh Van Nguyen, ${ }^{1,2,3}$ Janet Anderson, ${ }^{1}$ Christopher Vidal, ${ }^{1}$ Richard Fulton, ${ }^{1}$ Jamma Li, ${ }^{1,2,4}$ Suran L Fernando ${ }^{1,2,4}$
}

\begin{abstract}
Background: Screening for HLA-A ${ }^{\star} 31: 01 / H L A-B^{\star} 15: 02, H L A-B \star 57: 01$ and HLA-B ${ }^{\star 58: 01}$ is recommended in selected populations for prevention of carbamazepine, abacavir, and allopurinol-induced severe cutaneous adverse reactions (SCARs). Compared to conventional methods for detection of HLA alleles, PCR using a tag single nucleotide polymorphism (SNP) can be cost-effective, particularly where the surrogate marker SNP is in absolute linkage disequilibrium with the relevant HLA allele.
\end{abstract}

Objective: To determine guidelines for prevention of SCARs though predictive screening for the Australian Vietnamese population, the prevalence of four HLA alleles (HLA-A ${ }^{\star} 31: 01$, HLA-B ${ }^{\star}$ 15:02, HLA-B ${ }^{\star 57: 01}$ and HLA-B ${ }^{\star} 58: 01$ ) was examined. The utility of surrogate markers, rs2395029 and rs9263726, was investigated to predict for the presence of HLA-B ${ }^{\star 57: 01}$ and HLA-B*58:01, respectively.

Methods: Genotyping for specific HLA alleles was performed in 152 healthy Vietnamese living in Sydney using validated and established PCR-based methods. SNP genotyping was conducted using restriction-fragment-length-polymorphism analysis.

Results: rs2395029 and rs9263726 strongly correlated with HLA-B ${ }^{\star 57: 01}(\mathrm{k}=1, p<0.001)$ and HLA-B 58:01 ( $\mathrm{k}=0.9$, $p<0.001$ ) with $100 \%$ sensitivity and $100 \%$ negative predictive value for predicting the HLA-B*57:01 and HLA-B $58: 01$ carriers, respectively. A high prevalence of carriers of HLA-A*31:01 (3.29\%), HLA-B*15:02 (14.47\%), HLA-B^57:01 $(6.58 \%)$ and HLA-B ${ }^{\star 58: 01}(9.21 \%)$ was revealed.

Conclusions: Screening is recommended for these alleles in Australian Vietnamese prior to introducing relevant therapies. SNPs, rs2395029 and rs9263726, can be successfully used as surrogate markers for HLA-B ${ }^{\star} 57: 01$ and HLA-B58:01 in this population.

Key words: HLA, polymorphisms, abacavir, allopurinol, carbamazepine, Toxic Epidermal Necrolysis, Stevens-Johnson syndrome, Drug Rash with Eosinophilia and Systemic Symptoms, Vietnamese

\section{Citation:}

Nguyen, D. V., Anderson, J., Vidal, C., Fulton, R., Li, J., Fernando, S. L. (2022). The utility of surrogate markers in predicting HLA alleles associated with adverse drug reactions in Vietnamese. Asian Pac J Allergy Immunol, 40(2), 134-141. https://doi. org/10.12932/ap-170219-0493

\footnotetext{
Affiliations:

${ }^{1}$ ImmunoRheumatology Laboratory, New South Wales Health Pathology-North, Royal North Shore Hospital, Sydney, Australia Sydney Medical School-Northern, The University of Sydney, Sydney, Australia

Division of Allergy and Clinical Immunology, Vinmec International Hospital Times City, Hanoi, Vietnam

${ }^{4}$ Department of Clinical Immunology and Allergy, Royal North Shore Hospital, Sydney, Australia
}

Corresponding author:

Suran L Fernando

ImmunoRheumatology Laboratory, NSW Health Pathology-North, Royal North Shore Hospital,

Reserve Road, St Leonards. NSW 2065 Australia

E-mail: Suran.Fernando@health.nsw.gov.au

\section{Introduction}

The associations between immunologically mediated adverse drug reactions and certain Human Leukocyte Antigens (HLA) alleles have been well documented with an increasing body of information detailing genetic risk factors associated with these reactions. Commonly described associations include the HLA-B ${ }^{\star}$ 15:02 and HLA-A ${ }^{\star} 31: 01$ alleles with carbamazepine (CBZ)-induced severe cutaneous 
adverse drug reactions (SCARs), HLA-B ${ }^{\star 58: 01}$ with allopurinol-induced SCARs and HLA-B ${ }^{\star}$ 57:01 with abacavir (ABC)induced hypersensitivity reactions. In particular, a strong association between the presence of HLA-B ${ }^{\star}$ 15:02 and the development of CBZ-induced Stevens-Johnson syndrome (SJS)/Toxic Epidermal Necrolysis (TEN) has been reported, predominantly in Asian populations. ${ }^{1-3}$ In comparison, the HLA-A*31:01 allele has been associated with susceptibility to CBZ-induced SCARs in both Asian and Caucasian populations and is linked with all clinical phenotypes of SCARs including SJS/TEN and Drug Rash with Eosinophilia and Systemic Symptoms (DRESS)/Drug Induced Hypersensitivity Syndrome (DIHS). ${ }^{4-6}$ Unlike CBZ-induced SCARs, allopurinol-induced SCARs and ABC-induced hypersensitivity reactions are not phenotype and ethnicity specific. HLA-B ${ }^{\star}$ 58:01 is strongly associated with allopurinol-induced SJS/TEN and DRESS in many populations worldwide. ${ }^{7}$ The strong association of HLA-B*57:01 and ABC-induced hypersensitivity reactions has also been reported widely. ${ }^{8,9}$

To prevent drug-induced hypersensitivity reactions, screening for the appropriate HLA alleles prior to prescribing therapies is highly recommended in populations with high prevalence of these alleles. ${ }^{10}$ A number of studies have confirmed the benefit of HLA screening in the prevention of hypersensitivity reactions ${ }^{11-13}$ and its cost-effectiveness. ${ }^{14,15}$ However, the efficacy of HLA screening relies on the prevalence of these alleles in each population and the cost of the screening methods used. ${ }^{16}$ Therefore, identification of the atrisk population and knowledge of the associated frequency of these HLA alleles, combined with the development of a cost-effective screening method are crucial for implementation of predictive screening.

Currently, DNA-based HLA typing methods including polymerase chain reaction (PCR) utilizing sequence specific primers (PCR-SSP), PCR with sequence specific oligonucleotide probes (PCR-SSOP) and direct sequencing may be routinely employed to detect different HLA alleles at higher resolution level. These methods, however, are time-consuming, with a relatively slow turnaround time and are generally expensive ${ }^{17}$ and therefore less suitable for predictive screening prior to the implementation of therapies in the prevention of SCARs.

In searching for a simple, reliable and cost-effective method, a number of single nucleotide polymorphisms (SNPs) have been utilized to predict HLA alleles associated with adverse drug reactions, based on linkage disequilibrium (LD) and are useful as surrogate markers in specified populations. In 2006, de Bakker et al. reported complete LD between rs3909184 and HLA-B ${ }^{\star}$ 15:02 in 45 Han Chinese individuals and between rs1061235 and HLA-A*31:01 in 182 Caucasian, 44 Japanese and the 45 Han Chinese individuals, respectively. Additionally, complete LD between rs2395029 and HLA-B ${ }^{\star 57: 01}$ was found in the Caucasian cohort. ${ }^{18}$ Complete LD has also been reported for rs9263726, a SNP within the psoriasis susceptibility 1 candidate 1 (PSORS1C1) gene, with HLA-B ${ }^{\star}$ 58:01 in a Japanese cohort ${ }^{19}$ with a strong correlation also seen in a study of Eastern Chinese patients with SCARs. ${ }^{20}$
The accuracy, however, of these SNPs to predict particular HLA alleles varies across multiple populations. Notably, LD of rs9263726 with HLA-B ${ }^{\star 58: 01}$ has not been observed in an Australian cohort study. ${ }^{21}$ In contrast, LD between rs2395029 and HLA-B ${ }^{\star}$ 57:01 has been reported consistently across multiple populations. ${ }^{17,18}$ In consideration of this variability, it is necessary to evaluate the utility of these SNPs to predict risk alleles associated with adverse drug reactions in a population before introduction as a screening test.

In the Vietnamese population, HLA-B ${ }^{\star}$ 15:02 and HLA-B ${ }^{\star}$ 58:01 have been determined to increase susceptibility to carbamazepine and allopurinol induced SCARs. A case-control study in Vietnam in 2015, comprising 38 SCAR patients and $25 \mathrm{CBZ}$-tolerant controls, reported a strong association of HLA-B $^{\star}$ 15:02 and CBZ-induced SJS/TEN. ${ }^{22}$ Further extension of this study verified the association of HLA-B ${ }^{\star} 15: 02$ and CBZ-induced SJS/TEN in the Vietnamese patients and confirmed a strong association between the HLA-B ${ }^{\star}$ 58:01 allele and allopurinol-induced SCARs. Complete linkage was observed between the A allele of rs9263726 and HLA-B ${ }^{\star} 58: 01{ }^{23}$ This finding suggests that rs9263726 can be used to identify HLA-B ${ }^{\star} 58: 01$ carriers in the Vietnamese population. It should be noted however, that a case-control study is not the optimal design for evaluating association between a surrogate marker and its tagged HLA allele due to the possible confounding effect of a high prevalence of the HLA allele in the patient population and relatively low proportion in controls. A population based study would therefore constitute a preferable method for testing correlation between the relevant SNP and tagged HLA allele.

In Australia, the Vietnamese population is the ninth most prevalent racial group with a total of 294,798 residents (2016 Australian Census Data). Of these, 36\% are currently living in Sydney, New South Wales. ${ }^{24}$ Further, there is no data on the prevalence of HLA alleles associated with adverse drug reactions in this group. It would therefore be informative to assess the prevalence of these HLA alleles to assist with the formulation of predictive screening guidelines for the prevention of SCARs in Vietnamese in Australia.

This study was therefore initiated to determine the prevalence of four specific HLA alleles (HLA-A*31:01, HLA-B ${ }^{\star}$ 15:02, HLA-B ${ }^{\star} 57: 01$ and HLA-B ${ }^{\star 58: 01)}$ in Vietnamese in Australia and to evaluate the utility of the surrogate markers rs2395029 and rs9263726, to predict for the presence of HLA-B ${ }^{\star 57: 01}$ and HLA-B ${ }^{\star}$ 58:01 alleles respectively, in this population group.

\section{Methods \\ Sample population}

This study included 152, randomly recruited, healthy unrelated individuals of Vietnamese ancestry (82 males and 70 females, median age 30 years, range 18-83). Participants were currently living in Sydney, New South Wales, Australia and confirmed at least one biological parent to be of Vietnamese origin. The study was approved by Northern Sydney Health District HREC (HREC/15/HAWKE/86). Buccal swab samples were collected after obtaining informed consent. 


\section{DNA extraction}

Genomic DNA was extracted from buccal swabs (CEPTM Swab, Fitzco ${ }^{\oplus}$, Spring Park, MN, USA) as per manufacturer's instructions (AccuPrep Genomic DNA Extraction kit, Bioneer Corp, Daejeon, South Korea). The quality of genomic DNA was analysed by spectrophotometry using the Implen $\mathrm{Na}$ no-Photometer ${ }^{\circledast}$ yielding an average DNA concentration of 25 $\mathrm{ng} / \mu \mathrm{l}$ and an average of A260/280 ratio of 1.7.

\section{HLA genotyping}

HLA-A ${ }^{\star}$ 31:01/HLA-B ${ }^{\star}$ 15:02 alleles were detected using a multiplex real-time PCR assay with TaqMan ${ }^{\circledast}$ probes. ${ }^{25}$ Detection of HLA-B ${ }^{\star 58: 01 / 57: 01}$ alleles was performed using a multiplex real-time PCR assay with TaqMan ${ }^{\circledR}$ probes $^{26}$ followed by real-time PCR with SYBR ${ }^{\otimes}$ Green $^{27}$ for confirmation of HLA-B ${ }^{\star} 58: 01$ and a nested PCR protocol $^{28}$ for typing of HLA-B ${ }^{\star 57: 01}$.

\section{SNP genotyping}

Genotyping for SNPs (rs2395029 T>G and rs9263726 $\mathrm{G}>\mathrm{A}$ ) was performed using a PCR restriction fragment length polymorphism (RFLP) technique. Oligonucleotide primers were designed using IDT's PrimerQuest Tool incorporating Primer3 software (version 2.2.3) (Integrated DNA Technologies, Skokie, IL, USA) and synthesized by Bioneer (Bioneer Corp, Daejeon, South Korea). A 260 bp amplicon harbouring SNP rs926372621 (Forward primer: 5'-AAG CTC CAT CCA CCC CTG GT-3' and Reverse primer: 5'-ACA CAT TGG GTG GGG GAC AT-3') and a 576 bp amplicon harbouring
SNP rs2395029 (Forward primer: 5'-GTC TGG GCT CTT GGA AAT CA-3' and Reverse primer: 5'-GCT TCT ATA GGC ACA GCT CAA-3') were amplified, respectively. PCR was performed in a total volume of $25 \mu \mathrm{l}$ including $20 \mu \mathrm{l}$ of reaction mixture $\left[12.5 \mu \mathrm{l} \mathrm{GoTaq}{ }^{\oplus}\right.$ Green Master Mix (Promega Corp, Madison, WI, USA), $0.5 \mu \mathrm{l}$ primers (100 pmol/ $\mu \mathrm{l})$, PCR grade water to final volume] and $5 \mu \mathrm{l}$ of input genomic DNA. PCR conditions: Hot-start: $94^{\circ} \mathrm{C}$ for $10 \mathrm{~min}$, Denature: $94^{\circ} \mathrm{C}$ for $30 \mathrm{sec}$, Annealing: $65^{\circ} \mathrm{C}$ for $30 \mathrm{sec}$, Extension: $72^{\circ} \mathrm{C}$ for 30 sec, Final extension: $72^{\circ} \mathrm{C}$ for 5 min. Total cycles: 35 . For SNP rs9263726, a $25 \mu \mathrm{l}$ reaction volume consisting of PCR products and 2.5 units of restriction enzyme FokI (New England Biolabs, Ipswich, MA, USA) was incubated at $37^{\circ} \mathrm{C}$ for 60 $\mathrm{min}$, followed by enzyme inactivation at $65^{\circ} \mathrm{C}$ for $20 \mathrm{~min}$. The restriction enzyme cleaves the PCR product in the presence of the A allele, yielding fragments of 141 bp and 119 bp (Figure 1). For SNP rs2395029, a $25 \mu \mathrm{l}$ reaction volume consisting of the PCR products and 2.5 units of restriction enzyme XcmI (New England Biolabs, Ipswich, MA, USA) was incubated at $37^{\circ} \mathrm{C}$ for $60 \mathrm{~min}$, followed by enzyme inactivation at $65^{\circ} \mathrm{C}$ for $20 \mathrm{~min}$. The restriction enzyme cleaves the PCR product in the presence of the $\mathrm{T}$ allele, yielding fragments of $328 \mathrm{bp}$ and 248 bp (Figure 2).

Fragments of digested PCR products were visualised on $1.2 \%$ agarose gel $\left(\right.$ FlashGel $^{\circledR}$ Cassette, Lonza, Allendale, NJ, USA). Selected samples were confirmed by direct DNA sequencing using BigDye terminators v3.1 (Applied Biosystems, Waltham, WA, USA) (Figure 1A, B, C and Figure 2A, B, C).

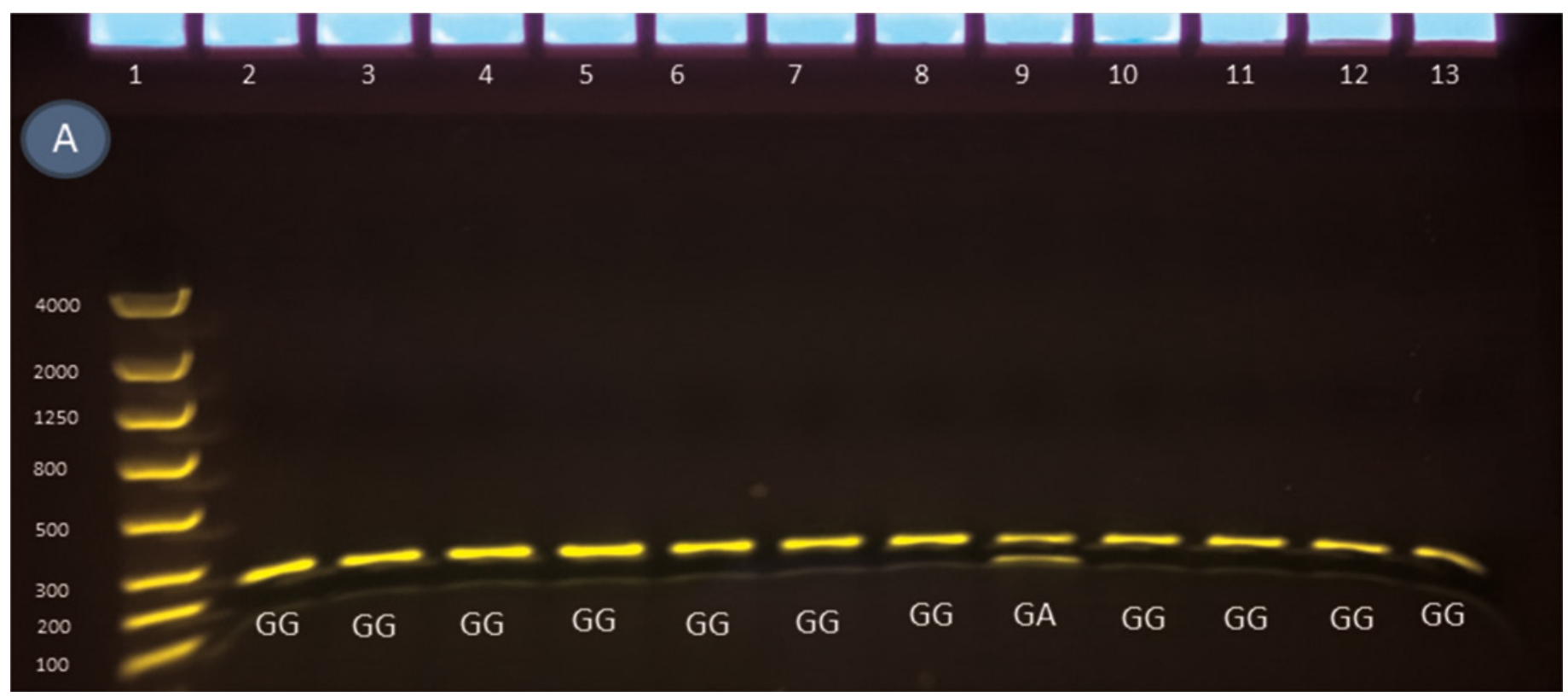

Figure 1. Demonstration of SNP rs9263726 genotyping.

(A) PCR product fragments on $1.2 \%$ agarose gel (FlashGel ${ }^{\circledR}$ Cassette, Lonza, Allendale, NJ, USA) after FokI digestion. Track 1 is the ladder. Track 9 is a heterozygous (GA) sample. 


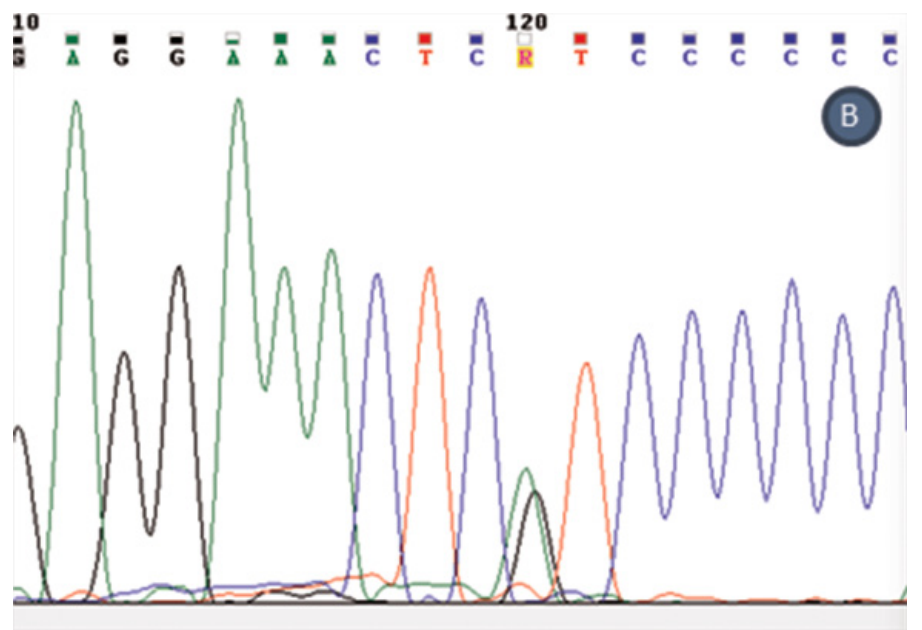

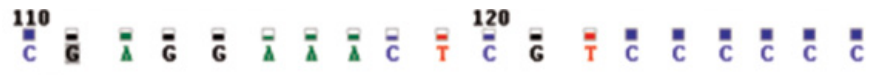

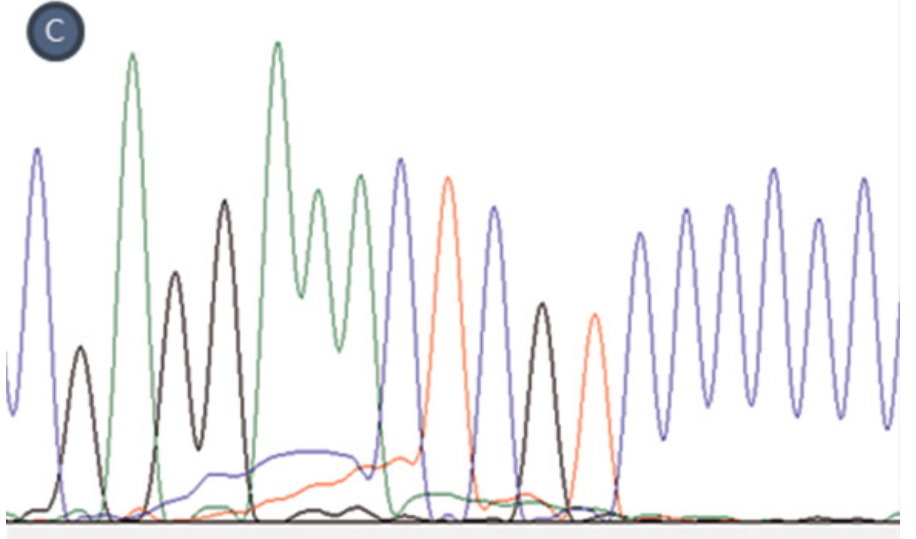

Figure 1. (Continued)

(B) shows the sequence of the SNP rs9263726 heterozygous (GA-R). (C) shows the sequence of the SNP rs9263726 wild type (GG).
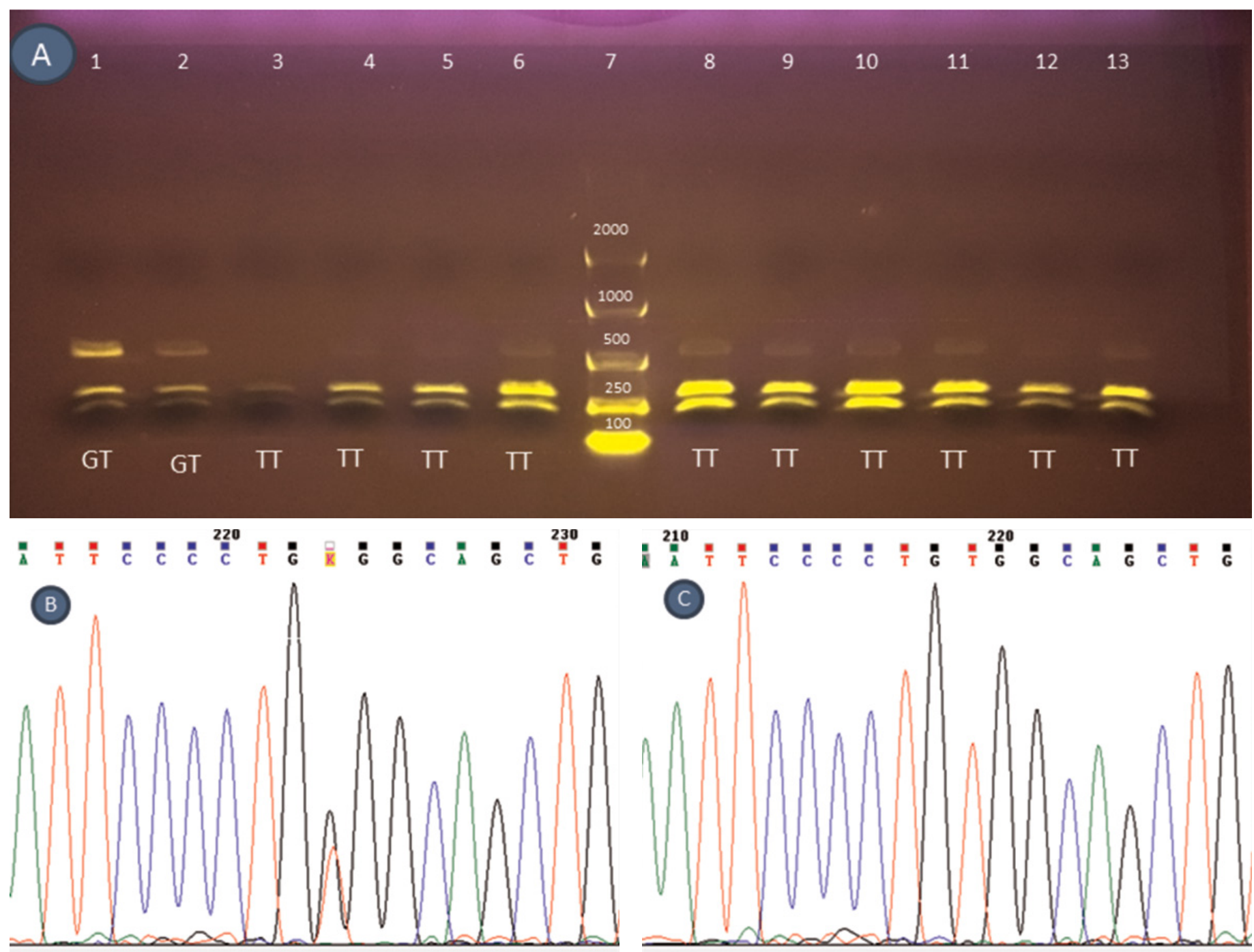

Figure 2. Demonstration of SNP rs2395029 genotyping.

(A) PCR product fragments on 1.2\% agarose gel (FlashGel ${ }^{\circledR}$ Cassette, Lonza, Allendale, NJ, USA) electrophoresis after XcmI digestion. Track 7 is the ladder. Tracks 1 and 2 are heterozygous (GT) samples. (B) shows the sequence of the heterozygous (GT-K) SNP rs2395029. (C) shows the sequence of the wild type (TT) of SNP rs2395029. 


\section{Data analysis}

Data was analysed using IBM SPSS Statistics software, version 22.0 (IBM SPSS, Armonk, NY, USA) including determination of the prevalence of each HLA allele (HLA-A*31:01, HLA-B $^{\star} 15: 02$, HLA-B ${ }^{\star}$ 57:01 and HLA-B $\left.{ }^{\star} 58: 01\right)$ and analysis of agreement between the surrogate marker SNPs (rs2395029 and rs9263726) and the relevant tagged HLA allele. The ability of each SNP (to potentially predict HLA-B*57:01 and HLA-B ${ }^{\star} 58: 01$, was additionally assessed by determination of sensitivity, specificity, positive predictive value (PPV) and negative predictive value (NPV). A web-based tool ${ }^{29}$ was used to test for Hardy-Weinberg Equilibrium (HWE) of each SNP. A $P$ value of $<0.05$ was considered as statistically significant.

\section{Results}

Prevalence of carriers for $H L A-A^{\star} 31: 01, H L A-B^{\star} 15: 02, H L A-$ $B \star 57: 01$ and $H L A-B \star 58: 01$ in the Vietnamese population in Australia

In this study, we successfully detected HLA-A*31:01, HLA-B $^{\star} 15: 02$, HLA-B ${ }^{\star 57: 01}$ and HLA-B ${ }^{\star 58: 01}$ in genomic DNA extracted from buccal swabs collected from healthy unrelated individuals of Vietnamese ancestry, using well validated real-time PCR methods. Results revealed the overall prevalence of carriers for HLA-A ${ }^{\star} 31: 01$ (3.29\%), HLA-B ${ }^{\star} 15: 02$ (14.47\%), HLA-B ${ }^{\star 57: 01}(6.58 \%)$ and HLA-B ${ }^{\star 58: 01}(9.21 \%)$ in this Vietnamese Australian cohort.

Comparison of these results with figures from studies in Vietnamese populations from other geographical regions, shows that the prevalence of HLA-A*31:01, HLA-B ${ }^{\star} 15: 02$, HLA-B ${ }^{\star} 57: 01$ and HLA-B ${ }^{\star}$ 58:01 carriers in our study group is similar to that reported in Vietnamese populations in Vietnam and the United States (Table 1).

In comparison to non-Vietnamese populations, the HLA$B^{\star}$ 15:02 and HLA-B ${ }^{\star}$ 58:01 alleles in particular were found with higher frequency in our cohort group than in a Caucasian population (Table 1). Additionally, the prevalence of HLA-B ${ }^{\star 57: 01}$ carriers was higher in the study population than in a Chinese population. The high prevalence of these alleles in our study cohort indicates that individuals of Vietnamese ancestry may be at high risk for carbamazepine and allopurinol-induced SCARs and for abacavir-induced hypersensitivity reactions.

\section{Correlation of rs2395029 $T>G$ and $H L A-B{ }^{\star 57: 01}$}

Of 152 samples, 10 which were identified as heterozygous carriers of the G allele of SNP rs2395029, were also positive for HLA-B ${ }^{\star} 57: 01$. The distribution of this SNP was in perfect HWE ( $\left.\mathrm{T}=0.97, \mathrm{G}=0.03, \chi^{2}=0.18, p>0.05\right)$. There was a perfect agreement between HLA-B ${ }^{\star} 57: 01$ and the SNP rs2395029 $(\mathrm{k}=1.0, p<0.001)$. The result is consistent with the findings across ethnic populations ${ }^{17,30}$ over the world, and indicates that SNP rs2395029 is an effective surrogate marker to detect HLA-B ${ }^{\star 57: 01}$ in individuals of Vietnamese ancestry, with calculated sensitivity, specificity, NPV and PPV all of $100 \%$ in our cohort group (Table 2).

Table 2. Concordance of HLA-B ${ }^{\star 57: 01}$ and SNP rs2395029.

\begin{tabular}{lccc} 
& \multicolumn{3}{c}{ SNP rs2395029 T>G genotype } \\
\cline { 2 - 4 } & TT & GT & GG \\
\hline HLA-B $* 57: 01$ Positive & 0 & 10 & 0 \\
HLA-B $* 57: 01$ Negative & 142 & 0 & 0 \\
Total & 142 & 10 & 0 \\
\hline
\end{tabular}

- Sensitivity: $100 \%, 95 \%$ CI [69.2 - 100\%]

- Specificity: $100 \%, 95 \%$ CI $[97.4-100 \%]$

- Positive predictive value: $100 \%$

- Negative predictive value: $100 \%$

Table 1. Prevalence of HLA alleles associated with SCARs in Vietnamese populations as compared to non-Vietnamese populations.

\begin{tabular}{|c|c|c|c|c|c|c|c|c|c|c|}
\hline & \multicolumn{2}{|c|}{$\begin{array}{c}\text { Vietnamese } \\
\text { Australia } \\
(\mathrm{n}=152)(\text { Present study) }\end{array}$} & \multicolumn{2}{|c|}{$\begin{array}{l}\text { Vietnamese } \\
\text { Vietnam } \\
(\mathbf{n}=170)^{34}\end{array}$} & \multicolumn{2}{|c|}{$\begin{array}{l}\text { Vietnamese } \\
\text { United States } \\
\left(\mathrm{n}=\mathbf{4 3 , 5 4 0 ) ^ { 3 5 }}\right.\end{array}$} & \multicolumn{2}{|c|}{$\begin{array}{c}\text { Caucasian } \\
\text { European, United States } \\
\left(\mathrm{n}=\mathbf{1 , 2 4 2 , 8 9 0 ) ^ { 3 5 }}\right.\end{array}$} & \multicolumn{2}{|c|}{$\begin{array}{c}\text { Chinese } \\
\text { United States } \\
(\mathbf{n}=99,672)^{35}\end{array}$} \\
\hline & $\begin{array}{l}\text { No. of } \\
\text { carriers }\end{array}$ & $\begin{array}{l}\text { Prevalence } \\
\%[95 \% \mathrm{CI}]^{\mathrm{a}}\end{array}$ & $\begin{array}{l}\text { Allele } \\
\text { frequency } \\
\text { (q) }\end{array}$ & $\begin{array}{c}\text { Estimated } \\
\text { prevalence } \\
\text { of carriers } \\
(\%)^{b}\end{array}$ & $\begin{array}{l}\text { Allele } \\
\text { frequency } \\
\text { (q) }\end{array}$ & $\begin{array}{c}\text { Estimated } \\
\text { prevalence } \\
\text { of carriers } \\
(\%)^{b}\end{array}$ & $\begin{array}{l}\text { Allele } \\
\text { frequency } \\
\text { (q) }\end{array}$ & $\begin{array}{c}\text { Estimated } \\
\text { prevalence } \\
\text { of carriers } \\
(\%)^{b}\end{array}$ & $\begin{array}{l}\text { Allele } \\
\text { frequency } \\
\text { (q) }\end{array}$ & $\begin{array}{l}\text { Estimated } \\
\text { prevalence } \\
\text { of carriers } \\
(\%)^{b}\end{array}$ \\
\hline 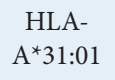 & 5 & $\begin{array}{c}3.29 \% \\
{[1.08 \%-7.51 \%]}\end{array}$ & 0.0210 & 4.20 & 0.0137 & 2.72 & 0.026977 & 5.29 & 0.024188 & 4.78 \\
\hline $\begin{array}{l}\text { HLA- } \\
\mathrm{B}^{*} 15: 02\end{array}$ & 22 & $\begin{array}{c}14.47 \% \\
{[9.30-21.09]}\end{array}$ & 0.1350 & 25.17 & 0.1383 & 27.13 & 0.000041 & $\approx 0$ & 0.064655 & 12.51 \\
\hline 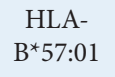 & 10 & $\begin{array}{c}6.58 \% \\
{[3.20 \%-11.33 \%]}\end{array}$ & 0.0290 & 5.72 & 0.0257 & 5.07 & 0.036453 & 7.13 & 0.005112 & 1.02 \\
\hline $\begin{array}{l}\text { HLA- } \\
\mathrm{B}^{\star} 58: 01\end{array}$ & 14 & $\begin{array}{c}9.21 \% \\
{[5.13 \%-14.97 \%]}\end{array}$ & 0.0650 & 12.16 & 0.0692 & 13.36 & 0.007264 & 1.44 & 0.083768 & 16.71 \\
\hline
\end{tabular}

a The Clopper-Pearson Exact method was used to calculate prevalence with a 95\% confidence interval (CI).

${ }^{b}$ The estimated prevalence of carriers was calculated based on HWE equation [prevalence of carriers $(\%)=\left[\left(q^{2}+2 p q\right) \times 100\right]$. 


\section{Correlation of rs9263726 G>A and HLA-B ${ }^{\star 58: 01}$}

Genotyping of SNP rs9263726, revealed 17 heterozygous carriers of the A allele, of which 15 samples were positive for HLA-B ${ }^{\star 58: 01}$. There was strong agreement between HLA-B ${ }^{\star} 58: 01$ and the SNP rs9263726 $(\mathrm{k}=0.9, p<0.001)$. The distribution of this SNP was in perfect HWE ( $\mathrm{G}=0.94$, $\left.\mathrm{A}=0.06, \chi^{2}=0.53, p>0.05\right)$. This finding indicates that SNP rs9263726 genotyping is an appropriate method for screening HLA-B ${ }^{\star 58: 01}$ carriers within the Australian Vietnamese population with high sensitivity, specificity, NPV and PPV again indicated (Table 3 ).

On further investigation, 2 of the 3 unmatched samples for HLA-B ${ }^{\star 58: 01}$ and SNP rs9263726 (one DNA sample was of insufficient quantity) were genotyped using Luminex SSO/SBT/SSP (Australian Red Cross Blood Service). Genotypes were revealed to be HLA-B ${ }^{\star} 40: 01 /$ HLA-B $^{\star} 40: 06$ and HLA-B ${ }^{\star} 07: 05-06 /$ HLA-B $^{\star}$ 18:01.

Table 3. Concordance of HLA-B ${ }^{\star 58: 01}$ and SNP rs9263726.

\begin{tabular}{lccc} 
& \multicolumn{3}{c}{ SNP rs9263726 G>A genotype } \\
\cline { 2 - 4 } & GG & GA & AA \\
\hline HLA-B ${ }^{\star} 58: 01$ Positive & 0 & 14 & 0 \\
HLA-B $* 58: 01$ Negative & 135 & 3 & 0 \\
Total & 135 & 17 & 0 \\
\hline
\end{tabular}

- Sensitivity: $100 \%, 95 \%$ CI [76.8 - 100\%]

- Specificity: $98.0 \%, 95 \%$ CI [93.8 - 99.6\%]

- Positive predictive value: $82.4 \%$, 95\% CI [60.4 - 93.5\%]

- Negative predictive value: $100 \%$

\section{Discussion}

With a total number of 294,798 , the Vietnamese population is the ninth largest racial group represented in Australia. ${ }^{24}$ In this study, we determined the prevalence of HLA alleles associated with adverse drug reactions in the Vietnamese population in Australia. The high prevalence of carriers for these alleles within the Vietnamese Australian population provides strong evidence for the utility of pharmacogenetic screening to aid in the prevention of CBZ, allopurinol and ABC-induced severe adverse reactions in this group. Furthermore, the presence of both HLA-B ${ }^{\star} 15: 02(14.47 \%)$ and HLA-A*31:01 (3.29\%) carriers in our sample cohort, supports the utility of a multiplex assay as a cost effective screening tool for both alleles associated with CBZ induced SCARs. This assay may additionally prove beneficial in other racial groups living in Australia, such as the Han Chinese, in which racial admixture results in maintenance of a high prevalence of $\mathrm{HLA}^{\star} \mathrm{B}^{\star}$ 15:02 and a rise in the frequency of HLA-A*31:01.

A number of recent studies have demonstrated that the cost-effectiveness of HLA screening relies on three crucial elements: the cost of treatment, the cost of the screening test and the prevalence of the culprit HLA allele in association with its PPV. ${ }^{15}$ Dong et al. indicated that HLA screening is likely to be most cost-effective in populations where HLA-B $^{\star} 15: 02$ prevalence is greater than $5 \%,{ }^{31}$ while screening for $\mathrm{HLA}-\mathrm{A}^{\star} 31: 01$ is indicated to be cost-effective in populations with an allelic prevalence greater than $2.5 \%{ }^{15}$
The current study reveals prevalence figures greater than these values in each case and thus supports cost effective screening for these markers in our study population. With respect to the HLA-B ${ }^{\star} 57: 01$ allele a high PPV of $47.9 \%$ has been reported for detection of hypersensitivity to abacavir. ${ }^{11}$ While the allelic prevalence is highest in white Caucasians (4-8\%) as compared to African Americans (2-3\%), Han Chinese and Africans $(<1 \%)^{32}$ the increasing rates of ethnic admixture and the increasing availability of predictive HLA-B ${ }^{\star 57: 01}$ screening assays, has resulted in recommendation of testing for all individuals. ${ }^{10}$ Again, screening is more likely to be cost effective in populations where HLA-B ${ }^{\star} 57: 01$ prevalence is high. ${ }^{14}$ The current study showed a prevalence of $6.58 \%$ for HLA-B ${ }^{\star} 57: 01$ in Vietnamese in Australia and therefore cost effective screening could be recommended for this allele prior to commencing abacavir therapy.

The association of rs9263726 and HLA-B ${ }^{\star 58: 01}$ demonstrated in this study is consistent with previous findings in a case-control study of allopurinol-induced SCARs conducted in Vietnam. ${ }^{23}$ This confirms the utility of the rs9263726 SNP as a surrogate marker for prediction of susceptibility to allopurinol-induced SCARs in individuals of Vietnamese ethnicity. The high (100\%) NPV of the test, relative to the presence of the HLA-B ${ }^{\star} 5801$ allele, minimizes the risk of exposing a potentially susceptible patient to a severe adverse drug reaction due to treatment with allopurinol. The presence of three HLA-B ${ }^{\star 58: 01}$ negative carriers possessing the $\mathrm{A}$ allele of rs9263726 and contributing to a slightly reduced PPV (82.4\%) agrees with data suggesting that rs9263726 is not in absolute LD with HLA-B ${ }^{\star 5801}$ across varying populations. ${ }^{21}$ Such false positive results however, do not increase the risk of adverse reactions, though ideally confirmatory testing for HLA-B ${ }^{\star} 58: 01$ should be performed in positive cases to avoid any unnecessary denial of allopurinol treatment.

The strong association of rs2395029 and HLA-B ${ }^{\star} 5701$ in this study indicates that this SNP may successfully be used as a marker to predict for the presence or absence of HLA-B ${ }^{\star 57: 01}$ carriage in Vietnamese individuals. Compared to other methods currently in use for HLA-B ${ }^{\star}$ 57:01 screening, SNP genotyping with a surrogate marker is a reliable, simple, accessible and affordable method. ${ }^{30}$ There are approximately 250,000 people living with HIV infection in Vietnam, many in rural and mountainous regions of the country. Antiretroviral coverage reached $50 \%$ by $2017 .{ }^{33}$ The U.S Department of Health and Human Services recommends abacavir based antiretroviral therapy as one of the first line options for treatment naïve patients. The availability of a cost effective screening method in Vietnam for the detection of HLA-B ${ }^{\star 57: 01}$ would not only prevent abacavir-induced hypersensitivity reactions but also allow for expansion of the availability of this antiretroviral drug. The ability, therefore, to perform onsite predictive testing for rs2395029 would be particularly attractive for remote settings in Vietnam.

In this study, successful genotyping for HLA using realtime PCR assay and SNPs using basic PCR-RFLP indicates that buccal swabs provide a sufficient DNA quality for implementing pharmacogenetic screening. Compared to blood collection, a buccal swab is non-invasive, more portable and more suitable for special populations such as children 
and elderly patients. Buccal swabs are also suitable for dealing with infectious samples, including HIV-infected samples.

In conclusion, the strong correlations between rs2395029

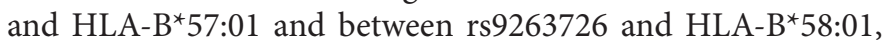
observed in this study, confirm the utility of these surrogate markers in the identification of HLA-B ${ }^{\star 57: 01}$ and HLA-B ${ }^{\star 58: 01}$ carriers. The availability of these simple and relatively less expensive methodologies may facilitate implementation of pharmacogenetic screening in Vietnamese populations in varying geographical locations and particularly in Vietnam where there is a lack of resources for HLA genotyping. The high prevalence of carriers for HLA-A*31:01, HLA $^{\star}{ }^{\star} 15: 02$, HLA $^{*}{ }^{\star}$ 57:01 and HLA-B ${ }^{\star 58: 01}$ in individuals of Vietnamese ancestry in Australia is consistent with figures noted for Vietnamese populations residing in other areas of the world and supports the implementation of HLA screening for the prevention of $\mathrm{CBZ}$, allopurinol and abacavir hypersensitivity in this population group.

\section{Acknowledgements}

We would like to acknowledge all the Vietnamese participants who generously donated samples for this study. We also thank Dr Ngo Quang, a primary care physician at Marrickville Medical Centre and Van Son Tran, a PhD candidate at University of Technology, Sydney for their assistance in the collection of samples. We thank Ms Anne' Proos and the staff in the Department of Molecular Genetics, NSW Health Pathology-North, Royal North Shore Hospital for the use of their facilities. We are also grateful to Australia Awards Scholarship for supporting Dr Dinh Van Nguyen and the NSW Health Pathology-North Immunology Trust Fund for funding this study.

\section{Conflicts of interest}

None

\section{Funding}

This work was supported by the NSW Health PathologyNorth Trust Fund. Dr Dinh Van Nguyen was supported by an Australia Awards Scholarship.

\section{References}

1. Chung WH, Hung SI, Hong HS, Hsih MS, Yang LC, Ho HC, et al. Medical genetics: a marker for Stevens-Johnson syndrome. Nature. 2004;428(6982):486.

2. Chang CC, Too CL, Murad S, Hussein SH. Association of HLA-B ${ }^{\star} 1502$ allele with carbamazepine-induced toxic epidermal necrolysis and Stevens-Johnson syndrome in the multi-ethnic Malaysian population. Int J Dermatol. 2011;50(2):221-4.

3. Nguyen DV, Chu HC, Nguyen DV, Phan MH, Craig T, Baumgart K, et al. HLA-B ${ }^{\star} 1502$ and carbamazepine-induced severe cutaneous adverse drug reactions in Vietnamese. Asia Pac Allergy. 2015;5(2):68-77.

4. Genin E, Chen DP, Hung SI, Sekula P, Schumacher M, Chang PY, et al. HLA-A ${ }^{\star 31: 01}$ and different types of carbamazepine-induced severe cutaneous adverse reactions: an international study and meta-analysis. Pharmacogenomics J. 2014;14(3):281-8.

5. Ozeki T, Mushiroda T, Yowang A, Takahashi A, Kubo M, Shirakata y, et al. Genome-wide association study identifies HLA-A ${ }^{\star 3101}$ allele as a genetic risk factor for carbamazepine-induced cutaneous adverse drug reactions in Japanese population. Hum Mol Genet. 2011;20(5):1034-41.
6. McCormack M, Alfirevic A, Bourgeois S, Farrell JJ, Kasperaviciute D, Carrington $\mathrm{M}$, et al. HLA-A ${ }^{*} 3101$ and carbamazepine-induced hypersensitivity reactions in Europeans. N Engl J Med. 2011;364(12): 1134-43.

7. Somkrua R, Eickman EE, Saokaew S, Lohitnavy M, Chaiyakunapruk N. Association of HLA-B ${ }^{\star} 5801$ allele and allopurinol-induced Stevens Johnson syndrome and toxic epidermal necrolysis: a systematic review and meta-analysis. BMC Med Genet. 2011;12:118.

8. Mallal S, Nolan D, Witt C, Masel G, Martin AM, Moore C, et al. Association between presence of HLA-B ${ }^{\star} 5701$, HLA-DR7, and HLA-DQ3 and hypersensitivity to HIV-1 reverse-transcriptase inhibitor abacavir. Lancet. 2002;359(9308):727-32.

9. Sousa-Pinto B, Pinto-Ramos J, Correia C, Goncalves-Costa G, Gomes L, Gil-Mata S, et al. Pharmacogenetics of abacavir hypersensitivity: A systematic review and meta-analysis of the association with HLA-B ${ }^{\star 57: 01 . ~ J ~ A l l e r g y ~ C l i n ~ I m m u n o l . ~ 2015 ; 136(4): 1092-4 . ~}$

10. Fricke-Galindo I, LLeena A, Lopez-Lopez M. An update on HLA alleles associated with adverse drug reactions. Drug Metabol Pers Ther. 2017;32(2):73-87.

11. Mallal S, Phillips E, Carosi G, Molina JM, Workman C, Tomazic J, et al. HLA-B ${ }^{\star} 5701$ screening for hypersensitivity to abacavir. N Engl J Med. 2008;358(6):568-79.

12. Ko TM, Tsai CY, Chen SY, Chen KS, Yu KH, Chu CS, et al. Use of HLA-B ${ }^{\star}$ 58:01 genotyping to prevent allopurinol induced severe cutaneous adverse reactions in Taiwan: national prospective cohort study. BMJ. 2015;351:h4848.

13. Chen P, Lin JJ, Lu CS, Ong CT, Hsieh PF, Yang CC, et al. Carbamazepine-induced toxic effects and HLA-B ${ }^{\star} 1502$ screening in Taiwan. N Engl J Med. 2011;364(12):1126-33.

14. Schackman BR, Scott CA, Walensky RP, Losina E, Freedberg KA, Sax PE. The cost-effectiveness of HLA-B ${ }^{\star} 5701$ genetic screening to guide initial antiretroviral therapy for HIV. AIDS. 2008;22(15):2025-33.

15. Plumpton CO, Roberts D, Pirmohamed M, Hughes DA. A sytematic review of economic evaluations of pharmacogenetic testing for prevention of adverse drug reactions. Pharmacoeconomics. 2016;34(8): 771-93.

16. Fernando SL, Broadfoot AJ. Prevention of severe cutaneous adverse drug reactions: the emerging value of pharmacogenetic screening. CMAJ. 2010;182(5):476-80.

17. He Y, Hoskins JM, Clark S, Campbell NH, Wagner K, Motsinger-Reif AA, et al. Accuracy of SNPs to predict risk of HLA alleles associated with drug-induced hypersensitivity events across racial groups. Pharmacogenomics. 2015;16(8):817-24.

18. de Bakker PI, McVean G, Sabeti PC, Miretti MM, Green T, Marchini J, et al. A high-resolution HLA and SNP haplotype map for disease association studies in the extended human MHC. Nat Genet. 2006;38(10):1166-72.

19. Tohkin M, Kaniwa N, Saito Y, Sugiyama E, Kurose K, Nishikawa J, et al. A whole-genome association study of major determinants for allopurinol-related Stevens-Johnson syndrome and toxic epidermal necrolysis in Japanese patients. Pharmacogenomics J. 2013;13(1):60-9.

20. Chen Z, Zhang S, Zhang J, Zhang Y, Xue L, Miao L. rs9263726 is a specific genetic marker for allopurinol-induced severe cutaneous adverse reactions in Chinese patients. Per Med. 2015;12(6):585-92.

21. Vidal C, Li J, Fulton R, Fernando SL. A polymorphism within the psoriasis susceptibility 1 candidate 1 (PSORS1C1) gene is not linked to HLA-B ${ }^{\star 58: 01}$ in an Australian cohort. Drug Metab Pharmacokinet. 2016;31(3):252-5.

22. Nguyen DV, Chu HC, Nguyen DV, Phan MH, Craig T, Baumgart K, et al. HLA-B ${ }^{\star} 1502$ and carbamazepine-induced severe cutaneous adverse drug reactions in Vietnamese. Asia Pac Allergy. 2015;5:68-77.

23. Nguyen DV, Chu HC, Vidal C, Nguyen NN, Do NTQ, Tran TTL, et al. Genetic Susceptibility to Carbamazepine and Allopurinol-Induced Severe Cutaneous Adverse Reactions in Vietnamese. J Allergy Clin Immunol. 2017;139(2):AB118.

24. Australian Bureau of Statistics [Internet]. Australia: General Community Profile; c2016 [cited 2019 Jan 29]. Available from: http://quickstats.census data.abs.gov.au/census_services/getproduct/census/2016/community profile/036

25. Nguyen DV, Vidal C, Chu HC, Do NTQ, Fulton R, Li J, et al. A novel multiplex polymerase chain reaction assay for detection of both HLA-A $^{*} 31: 01 /$ HLA $^{*}$ 15:02 alleles, which confer susceptibility to carbamazepine-induced severe cutaneous adverse reactions. HLA. 2017;90(6):335-42. 
26. Nguyen DV, Vidal C, Li J, Fulton RB, Fernando SL. Validation of a rapid test for HLA-B ${ }^{\star} 58: 01 / 57: 01$ allele screening to detect individuals at risk for drug-induced hypersensitivity. Pharmacogenomics. 2016;17(5): 473-80.

27. Nguyen DV, Vidal C, Chu HC, Fulton RB, Li J, Fernando SL. Validation of a rapid, robust and inexpensive screening method for detection of HLA-B $\star^{58: 01}$ in prevention of allopurinol-induced severe cutaneous adverse reactions. Allergy Asthma Immun. 2017;9(1):79-84.

28. Cascella R, Strafella C, Ragazzo M, Zampatti S, Borgiani P, Gambardella S, et al. Direct PCR: a new pharmacogenetic approach for

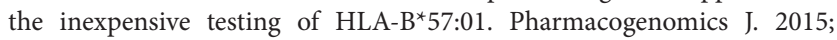
15(2):196-200.

29. Rodriguez S, Gaunt TR, Day IN. Hardy-Weinberg equilibrium testing of biological ascertainment for Mendelian randomization studies. Am J Epidemiol. 2009;169(4):505-14.

30. Rodriguez-Novoa S, Cuenca L, Morella J, Cordoba M, Blanco F, Jiminez-Nacher I, et al. Use of the HCP5 single nucleotide polymorphism to predict hypersensitivity reactions to abacavir: correlation with HLA-B ${ }^{\star 5701 . ~ J ~ A n t i m i c r o b ~ C h e m o t h e r . ~ 2010 ; 65(8): 1567-9 . ~}$
31. Dong D, Sung S, Finkelstein EA. Cost-effectiveness of HLA-B ${ }^{\star} 1502$ genotyping in adult patients with newly diagnosed epilepsy in Singapore. Neurology. 2012;79(12):1259-67.

32. Gonzalez-Galarza FF, Takeshita LY, Santos EJ, Kempson F, Maia MH, da Silva AL et al. Allele frequency net 2015 update: new features for HLA epitopes, KIR and disease and HLA adverse drug reaction associations, Nucleic Acids Res. 2015;43:D784-8.

33. UNAIDS [Internet]. Geneva: Viet Nam; c2017 [cited 2019 Jan 29]. Available from: http://www.unaids.org/en/regionscountries/countries/ vietnam

34. Hoa BK, Hang NT, Kashiwase K, Ohashi J, Lien LT, Horie T, et al. HLA-A, -B, -C, -DRB1 and -DQB1 alleles and haplotypes in the Kinh population in Vietnam. Tissue Antigens. 2008;71(2):127-34.

35. Gragert L, Madbouly A, Freeman J, Maiers M. Six-locus high resolution HLA haplotype frequencies derived from mixed-resolution DNA typing for the entire US donor registry. Hum Immunol. 2013;74(10):1313-20. 